\title{
A mild and convenient oxidative transformation of aldehydes to methyl esters using iodosobenzene in combination with $\mathrm{KBr}$
}

\author{
Loveena Arora $^{a}$, Richa Prakash ${ }^{b^{*}}$, Om Prakash , and Nitya Sharma $^{\mathrm{b}}$ \\ ${ }^{a}$ Department of Chemistry, National Institute of Technology, Kurukshetra, Haryana, India \\ ${ }^{b}$ Department of Chemistry, Kurukshetra University, Kurukshetra, Haryana, India \\ ${ }^{c}$ Madhav University, Abu road, Sirohi, Rajasthan, 307026, India \\ E-mail: prakashricha@rediffmail.com
}

DOI: http://dx.doi.org/10.3998/ark.5550190.p009.267

\begin{abstract}
Oxidation of various aliphatic and aromatic aldehydes with iodosobenzene in combination with potassium bromide in aqueous methanol offers a mild and convenient method for their conversion into the corresponding methyl esters. The method is not straightforward for the synthesis of heteroaryl esters.
\end{abstract}

Keywords: Organohypervalent iodine(III) reagents, iodosobenzene, oxidation, methyl esters

\section{Introduction}

Oxidative transformation of aldehydes (1) into esters (2) is a very useful reaction in organic synthesis. A number of methods using various reagents have been reported to bring about this transformation (Scheme 1). ${ }^{1-9}$ However, many of these procedures suffer from several limitations such as involvement of toxic heavy metal oxidants, expensive catalyst, large excess of reagents, and dry solvent in inert atmosphere. Furthermore, the requirement of poisonous ${ }^{3}$ and polluting reagents, mediators, and cocatalysts along with long reaction times and drastic reaction conditions causes severe economic and operational problems. Thus, alternative methods for this transformation are desirable.

Organohypervalent iodine reagents ${ }^{10-19}$ have been increasingly used in contemporary organic synthesis. In particular, organoiodine(III) reagents, namely (diacetoxyiodo)benzene, ${ }^{20}$ bis(trifluoroacetoxy)iodobenzene, ${ }^{21} \quad$ [hydroxy(tosyloxy)iodobenzene $]^{22}$ and iodosobenzene $(\mathrm{IOB})^{23}$ have emerged as versatile oxidizing agents. Organoiodine(III) reagents in combination with other reagents have also shown interesting applications in organic synthesis. For example, Kita et al. ${ }^{24,25}$ have reported that iodosobenzene in combination with $\mathrm{KBr}$ (system $i$ ) in aqueous methanol can be employed for the oxidative transformation of alcohols into methyl esters. This reaction has been suggested to proceed through the intermediacy of aldehyde, but these were not isolated. Based on these observations, it was considered worthwhile to examine the oxidation of 
aldehydes under similar conditions with a view to develop a facile method for the conversion of aldehydes into esters. Consequently, we report herein a new application of the system $i$ for the oxidative conversion of various aldehydes into methyl esters.

\section{Results and Discussion}

First, we attempted the oxidation of benzaldehyde (1d) with 1.1 equivalent of IOB and $\mathrm{KBr}(0.2$ equivalent) in aqueous methanol according to the reported conditions. ${ }^{24,25}$ However, to our surprise the reaction required more than two days for completion as compared to six hours for conversion of benzyl alcohol to methyl benzoate. The situation was worse when other, substituted benzaldehydes, such as $p$-nitro and $p$-methoxy derivatives were subjected to similar oxidation, most of the starting aldehydes being recovered even after stirring the reaction mixture for 50 hours. After making several attempts, it was found that using a larger amount of methanol leads to considerable increase in conversion of aldehydes 1 to methyl esters 2 . So it became necessary to use methanol in larger amount (see experimental) for all cases.

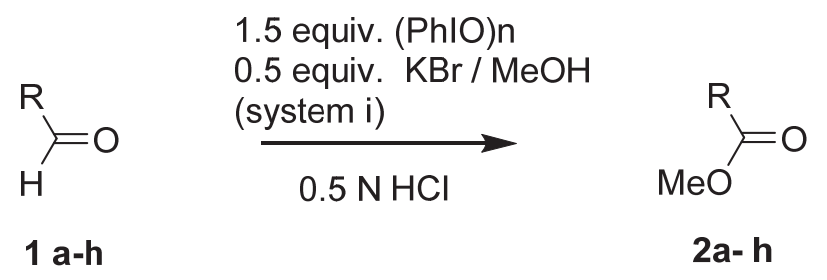

\section{Scheme 1}

After optimizing the reaction conditions for benzaldehyde (1d), the generality of this methodology was tested by carrying out the oxidation of various aliphatic (1a-c) and aromatic (1e-h) aldehydes. The method was successful in all cases, and the results are summarized in Table 1.

As expected, the reactions of aromatic aldehydes were slower than aliphatic aldehydes. Further, among aromatic aldehydes, the reactions of those with electron-donating groups at a para position gave better results.

When applied to heteroaryl aldehydes, the method was not straightforward in all cases. For example, this approach smoothly converted oxygen and sulfur containing heteroaryl aldehydes such as 2-furancarboxaldehyde (1i), 2-thiophenecarboxaldehyde (1j) and 2,5thiophenedicarboxaldehyde (1k) into the corresponding methyl esters $\mathbf{2} \mathbf{i}-\mathbf{2} \mathbf{k}$. On the other hand, nitrogen-containing heteroaryl aldehydes such as 4-pyrazolecarboxaldehydes on oxidation using system $i$ did not afford the desired esters. Instead, a novel $\mathrm{C}-\mathrm{C}$ bond cleavage was observed. The detailed investigations on the oxidation of various heteroaryl aldehydes are under progress and will be published elsewhere. 
Table 1. Oxidative esterification of aldehydes (1a-h) with system $i$

\begin{tabular}{|c|c|c|c|c|c|}
\hline Entry & Aldehyde 1 & Product 2 & $\begin{array}{l}\text { Time } \\
\text { (h) }\end{array}$ & $\begin{array}{c}\mathrm{Mp} \\
\text { (Lit. } \mathrm{mp} \text { ) }{ }^{\circ} \mathrm{C}\end{array}$ & $\begin{array}{c}\text { Yield }^{\mathrm{c}} \\
(\%)\end{array}$ \\
\hline 1 & $\mathrm{CH}_{3}\left(\mathrm{CH}_{2}\right)_{4} \mathrm{CHO}, \mathbf{1 a}$ & $\mathrm{CH}_{3}\left(\mathrm{CH}_{2}\right)_{4} \mathrm{COOMe}, \mathbf{2 a}$ & 2 & liquid $^{\mathrm{a}}$ & 66 \\
\hline 2 & $\mathrm{CH}_{3}\left(\mathrm{CH}_{2}\right)_{6} \mathrm{CHO}, \mathbf{1 b}$ & $\mathrm{CH}_{3}\left(\mathrm{CH}_{2}\right)_{6} \mathrm{COOMe}, \mathbf{2 b}$ & 2 & liquid $^{\mathrm{a}}$ & 70 \\
\hline 3 & $\mathrm{CH}_{3}\left(\mathrm{CH}_{2}\right)_{7} \mathrm{CHO}, \mathbf{1 c}$ & $\mathrm{CH}_{3}\left(\mathrm{CH}_{2}\right)_{7} \mathrm{COOMe}, \mathbf{2 c}$ & 2 & liquid $^{\mathrm{a}}$ & 68 \\
\hline 4 & $\mathrm{C}_{6} \mathrm{H}_{5} \mathrm{CHO}, \mathbf{1 d}$ & $\mathrm{C}_{6} \mathrm{H}_{5} \mathrm{COOMe}, \mathbf{2 d}$ & 5 & liquid $^{\mathrm{a}}$ & 90 \\
\hline 5 & 4- $\mathrm{ClC}_{6} \mathrm{H}_{4} \mathrm{CHO}, \mathbf{1 e}$ & 4-ClC ${ }_{6} \mathrm{H}_{4} \mathrm{COOMe}, \mathbf{2 e}$ & 6 & $42-44^{\mathrm{b}}(44)^{27}$ & 60 \\
\hline 6 & $4-\mathrm{MeC}_{6} \mathrm{H}_{4} \mathrm{CHO}, \mathbf{1 f}$ & 4- $\mathrm{MeC}_{6} \mathrm{H}_{4} \mathrm{COOMe}, \mathbf{2 f}$ & 4 & $34^{\mathrm{b}}(33-36)^{27}$ & 75 \\
\hline 7 & 4- $\mathrm{MeOC}_{6} \mathrm{H}_{4} \mathrm{CHO}, \mathbf{1 g}$ & 4- $\mathrm{MeOC}_{6} \mathrm{H}_{4} \mathrm{COOMe}, \mathbf{2 g}$ & 4 & $49^{\mathrm{b}}(49-51)^{27}$ & 65 \\
\hline 8 & $4-\mathrm{O}_{2} \mathrm{NC}_{6} \mathrm{H}_{4} \mathrm{CHO}, \mathbf{1 h}$ & $4-\mathrm{O}_{2} \mathrm{NC}_{6} \mathrm{H}_{4} \mathrm{COOMe}, \mathbf{2 h}$ & 20 & $94-96^{\mathrm{b}}(96)^{27}$ & 55 \\
\hline 9 & 1i & $2 \mathbf{i}$ & 2 & liquid $^{\mathrm{a}}$ & 68 \\
\hline 10 & $\mathbf{1 j}$ & $2 \mathbf{j}$ & 3 & liquid $^{\mathrm{a}}$ & 72 \\
\hline 11 & $1 \mathrm{k}$ & $2 k$ & 4 & $\begin{array}{c}83-84^{\mathrm{b}} \\
(82-83)^{28}\end{array}$ & $74^{\mathrm{d}}$ \\
\hline
\end{tabular}

${ }^{a}$ Purity of the liquid products was confirmed by tlc and satisfactory elemental analysis $(C, H)$.

b All these products were finally purified by recrystallization by using minimum amount of methanol. For products $\mathbf{2 e}, \mathbf{2} \mathbf{f}$ and $\mathbf{2 g}$, the hot solution was slowly cooled to room temperature and then left in refrigerator for few hours.

${ }^{\mathrm{c}}$ Yield of the isolated pure product w.r.t. aldehyde $\mathbf{1}$.

${ }^{\mathrm{d}}$ The quantity of the reagents and methanol was doubled.

To study the further scope of this approach, we also carried out oxidation of $p$ chlorobenzaldehyde using two other alcohols, namely ethanol and 2-propanol. It was observed that the use of methanol is critical for the success of this oxidative transformation as in these 
other cases we were unable to isolate the corresponding ester in any detectable amount. Of course, iodosobenzene was quantitively reduced to iodobenzene.

A plausible mechanism of this IOB mediated conversion of $\mathbf{1}$ to $\mathbf{2}$, outlined in Scheme 2, is analogous to that suggested by Kita et al. for the oxidative conversion of alcohols into esters. Reactive I(III) species 3, which is generated in situ by depolymerization of iodosobenzene with $\mathrm{KBr}$, reacts with hemiacetal $4\left(\mathrm{R}^{\prime}=\mathrm{Me}\right)$ to give $\mathrm{O}-\mathrm{I}(\mathrm{III})$ intermediate 5. Subsequent reductive elimination of iodobenzene gives the ester 2 . The alternative possibility - that the carboxylic acid, 7, formed by the oxidation of aldehyde 1 through $4\left(R^{\prime}=H\right)$ and then intermediate 6 , undergoes esterification - is less likely under aqueous conditions. When benzoic acid was used instead of benzaldehyde under identical conditions, no trace of methyl benzoate could be isolated.

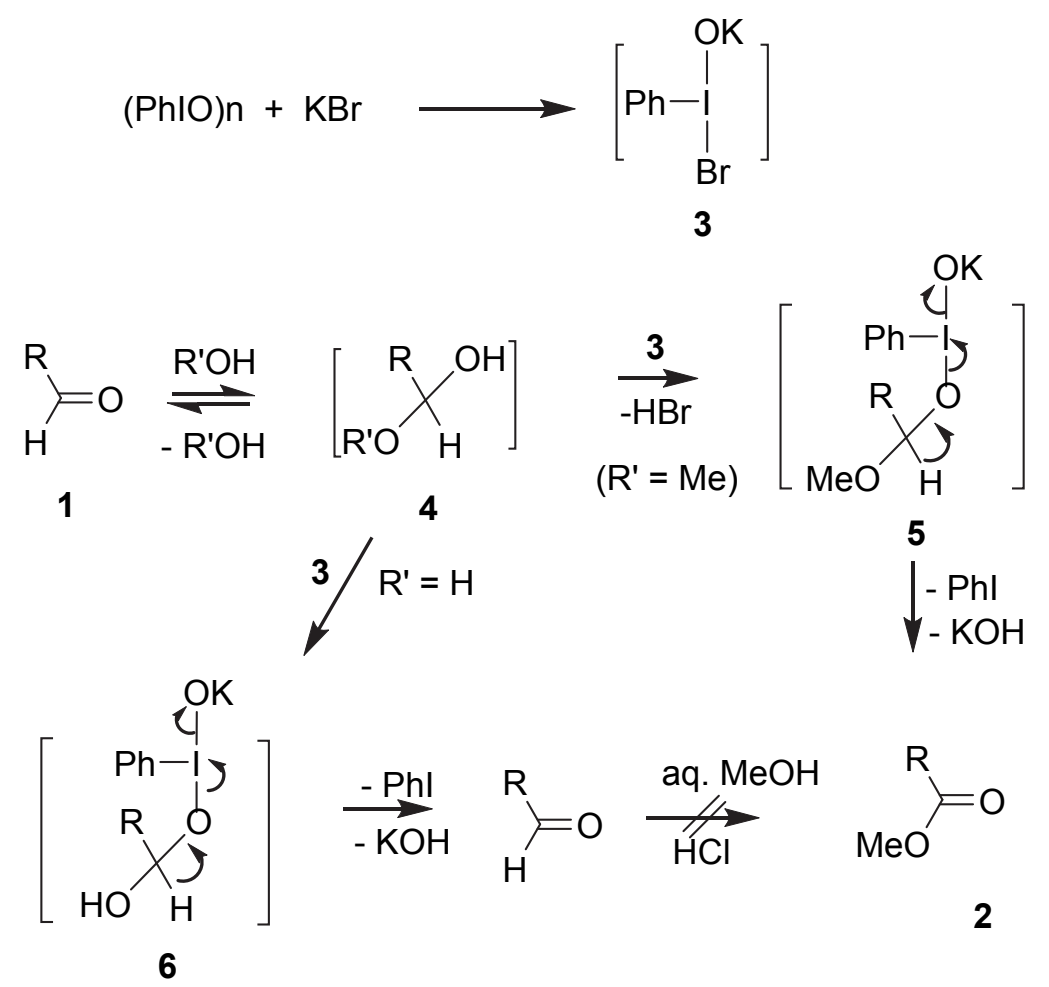

\section{Scheme 2}

\section{Conclusions}

The present study offers a new and convenient method for the conversion of a variety of aldehydes into the corresponding methyl esters. The method has advantages over the existing methods for the following reasons:

(i) The experimental procedure is very simple and conditions are mild. 
(ii) It does not involve expensive and toxic transition metal oxidants.

(iii) It is environmentally acceptable.

(iv) The iodobenzene produced quantitatively by the reductive elimination can be recycled to IOB.

(v) Examples covered in the present work illustrate its utility for the synthesis of three aliphatic, four aromatic and three heteroaromatic esters; synthetic application to other heteroaromatic and complex esters are to be investigated.

\section{Experimental Section}

General. Melting points were taken in open capillaries in a sulfuric acid bath and are uncorrected. ${ }^{1} \mathrm{H}$ NMR spectra were recorded on a Brucker $400-\mathrm{MHz}$ instrument using TMS as an internal standard. The methyl esters 2 were confirmed by comparison with literature melting points and ${ }^{1} \mathrm{H}$ NMR data. Column chromatography was performed using 63-200 mesh silica gel (Merck).

Iodosobenzene was prepared according to the literature procedure ${ }^{21,22,23}$ by hydrolysis of commercially available (diacetoxyiodo)benzene.

General procedure for $\mathbf{2 a - 2 j}$. To a magnetically stirred solution of aldehyde (1 mmol), and potassium bromide $(0.5 \mathrm{mmol})$ in $\mathrm{MeOH}(10 \mathrm{~mL})$ was added dropwise $0.5 \mathrm{~N} \mathrm{HCl}(2 \mathrm{~mL})$ followed by iodosobenzene $(1.5 \mathrm{mmol} ; 330 \mathrm{mg})$ and the solution stirred. Progress of the reaction was monitored by TLC. After completion of reaction, the mixture was diluted with $\mathrm{H}_{2} \mathrm{O}$ followed by extraction using $\mathrm{CH}_{2} \mathrm{Cl}_{2}(2 \times 10 \mathrm{~mL})$. The combined organic phase was dried over anhydrous $\mathrm{Na}_{2} \mathrm{SO}_{4}$ and solvent was removed by distillation to give crude product, which was purified by column chromatography (petroleum ether and EtOAc). Purity of the liquid products was checked by tlc and satisfactory elemental analysis $(\mathrm{C}, \mathrm{H})$. All the solid products were finally purified by recrystallization by using minimum amount of methanol. For the products $\mathbf{2 e}, \mathbf{2} \mathbf{f}$ and $\mathbf{2 g}$, the hot methanolic solution was slowly cooled to room temperature and then left in refrigerator for few hours.

Methyl hexanoate (2a). IR ( $v_{\max }$, neat): $1723 \mathrm{~cm}^{-1}(\mathrm{C}=\mathrm{O}),{ }^{1} \mathrm{H}$ NMR $\left(\mathrm{CDCl}_{3}, \delta\right): 3.66(\mathrm{~s}, 3 \mathrm{H})$, 2.30 (t, J 8 Hz. 2H), 1.66 (m, 2H), 1.33 (m, 2H), 0.89 (m, 5H).

Methyl octanoate (2b). IR ( $v_{\max }$, neat): $1722 \mathrm{~cm}^{-1}(\mathrm{C}=\mathrm{O}),{ }^{1} \mathrm{H} \mathrm{NMR}\left(\mathrm{CDCl}_{3}, \delta\right): 3.64(\mathrm{~s}, 3 \mathrm{H})$, $2.28(\mathrm{t}, J 7 \mathrm{~Hz}, 2 \mathrm{H}), 1.60(\mathrm{~m}, 2 \mathrm{H}), 1.27(\mathrm{~m}, 8 \mathrm{H}), 0.87(\mathrm{t}, 3 \mathrm{H})$.

Methyl nonanoate (2c). IR ( $v_{\max }$, neat): $1724 \mathrm{~cm}^{-1}(\mathrm{C}=\mathrm{O}),{ }^{1} \mathrm{H} \mathrm{NMR}\left(\mathrm{CDCl}_{3}, \delta\right): 3.82$ (s, 3H), 2.1 (t, $J 6 \mathrm{~Hz}, 2 \mathrm{H}), 1.42(\mathrm{~m}, 2 \mathrm{H}), 1.10-1.22(\mathrm{~m}, 10 \mathrm{H}), 0.85(\mathrm{t}, 3 \mathrm{H})$.

Methyl benzoate (2d). IR ( $v_{\max }$, neat): $1728 \mathrm{~cm}^{-1}(\mathrm{C}=\mathrm{O}),{ }^{1} \mathrm{H} \mathrm{NMR}\left(\mathrm{CDCl}_{3}, \delta\right): 3.90(\mathrm{~s}, 3 \mathrm{H}$, $\mathrm{OCH}_{3}$ ), 7.32-7.49 (m, 3H, Ar-H), 7.99-8.02 (d, 2H, Ar-H). 
Methyl 4-chlorobenzoate (2e). $\mathrm{Mp} 42-44{ }^{\circ} \mathrm{C}$ (lit. $\left.\mathrm{mp} 44{ }^{\circ} \mathrm{C}\right)^{27}$, IR ( $\left.v_{\max }, \mathrm{KBr}\right): 1715 \mathrm{~cm}^{-1}$ $(\mathrm{C}=\mathrm{O}),{ }^{1} \mathrm{H} \mathrm{NMR}\left(\mathrm{CDCl}_{3}, \delta\right): 3.89\left(\mathrm{~s}, 3 \mathrm{H}, \mathrm{OCH}_{3}\right), 7.41-7.59(\mathrm{~d}, 2 \mathrm{H}, J 8.3 \mathrm{~Hz}, \mathrm{Ar}-\mathrm{H}), 7.89-8.02$ (d, $2 \mathrm{H}, J 8.3 \mathrm{~Hz}, \mathrm{Ar}-\mathrm{H})$.

Methyl 4-methylbenzoate (2f). Mp $34{ }^{\circ} \mathrm{C}$ (lit. mp 33-36 $\left.{ }^{\circ} \mathrm{C}\right)^{27}, \mathrm{IR}\left(v_{\max }\right.$, neat): $1728 \mathrm{~cm}^{-1}(\mathrm{C}=\mathrm{O})$, ${ }^{1} \mathrm{H} \mathrm{NMR}\left(\mathrm{CDCl}_{3}, \delta\right): 2.41\left(\mathrm{~s}, 3 \mathrm{H}, \mathrm{CH}_{3}\right), 3.92\left(\mathrm{~s}, 3 \mathrm{H}, \mathrm{OCH}_{3}\right), 7.32-7.49(\mathrm{~d}, 2 \mathrm{H}, J 8.0 \mathrm{~Hz}, \mathrm{Ar}-\mathrm{H})$, 7.99-8.02 (d, 2H, J 8.0 Hz, Ar-H).

Methyl 4-methoxybenzoate (2g). Mp $49^{\circ} \mathrm{C}$ (lit. mp 49-51 $\left.{ }^{\circ} \mathrm{C}\right)^{27}$, IR ( $v_{\text {max }}$, neat): $1730 \mathrm{~cm}^{-1}$ $(\mathrm{C}=\mathrm{O}),{ }^{1} \mathrm{H} \mathrm{NMR}\left(\mathrm{CDCl}_{3}, \delta\right): 3.89\left(\mathrm{~s}, 3 \mathrm{H}, \mathrm{OCH}_{3}\right), 3.92\left(\mathrm{~s}, 3 \mathrm{H}, \mathrm{OCH}_{3}\right), 7.00-7.15(\mathrm{~d}, 2 \mathrm{H}, J 8.2 \mathrm{~Hz}$, Ar-H), 7.29-7.40 (d, 2H, J 8.2 Hz, Ar-H).

Methyl 4-nitrobenzoate (2h). Mp 94-96 ${ }^{\circ} \mathrm{C}\left(\text { lit. } \mathrm{mp} 96{ }^{\circ} \mathrm{C}\right)^{27}, \mathrm{IR}\left(v_{\max }, \mathrm{KBr}\right): 1705 \mathrm{~cm}^{-1}(\mathrm{C}=\mathrm{O})$, ${ }^{1} \mathrm{H}$ NMR $\left(\mathrm{CDCl}_{3}, \delta\right): 3.99$ (s, 3H, $\left.\mathrm{OCH}_{3}\right), 7.61-7.82$ (d, 2H, J 8.0 Hz, Ar-H), 8.01-8.02 (d, 2H, $8.0 \mathrm{~Hz}, \mathrm{Ar}-\mathrm{H})$.

Methyl 2-furancarboxylate (2i). IR ( $v_{\max }$, neat): $1770 \mathrm{~cm}^{-1}(\mathrm{C}=\mathrm{O}),{ }^{1} \mathrm{H} \mathrm{NMR}\left(\mathrm{CDCl}_{3}, \delta\right): 3.99$ (s, $\left.3 \mathrm{H}, \mathrm{OCH}_{3}\right), 7.15$ (d, 1H, J 4.55 Hz), $7.88(\mathrm{~d}, 1 \mathrm{H}, J 3.70 \mathrm{~Hz}) 6.68(\mathrm{~m}, 1 \mathrm{H}, J 1.30 \mathrm{~Hz})$.

Methyl 2-thiophenecarboxylate (2j). IR ( $v_{\max }$, neat): $1768 \mathrm{~cm}^{-1}(\mathrm{C}=\mathrm{O}),{ }^{1} \mathrm{H} \mathrm{NMR}\left(\mathrm{CDCl}_{3}, \delta\right)$ : $3.93\left(\mathrm{~s}, 3 \mathrm{H}, \mathrm{OCH}_{3}\right), 7.80(\mathrm{~d}, 1 \mathrm{H}, J 3.70 \mathrm{~Hz}), 7.10(\mathrm{~m}, 1 \mathrm{H}, J 1.30 \mathrm{~Hz}) 7.55$ (d, 1H, J 5.00 Hz).

Dimethyl 2,5-thiophenedicarboxylate (2k). Mp $83-84^{\circ} \mathrm{C}$ (lit. $\left.\mathrm{mp} 82-83{ }^{\circ} \mathrm{C}\right)^{28}$, IR ( $v_{\max }$, neat): $1766 \mathrm{~cm}^{-1}(\mathrm{C}=\mathrm{O}),{ }^{1} \mathrm{H}$ NMR $\left(\mathrm{CDCl}_{3}, \delta\right): 3.93\left(\mathrm{~s}, 6 \mathrm{H}, \mathrm{OCH}_{3}\right), 7.75(\mathrm{~s}, 2 \mathrm{H})$. This was prepared according to general procedure, but the quantity of the reagents and solvent $\mathrm{MeOH}$ was doubled and the reaction mixture was allowed to stir at $\mathrm{rt}$ for $4 \mathrm{~h}$.

\section{References and Notes}

1. Stevens, R. V.; Chapman, K. T.; Stubbs, C.A.; Tam, W. W.; Albizati, K. F. Tetrahedron Lett. 1982, 23,4647 .

http://dx.doi.org/10.1016/S0040-4039(00)85677-4

2. Gopinath, R.; Patel, B. K. Org. Lett. 2002, 2, 577.

http://dx.doi.org/10.1021/o1990383+

3. Sundararaman, P.; Walker, E. C.; Djerassi, C. Tetrahedron Lett. 1978, 1627.

http://dx.doi.org/10.1016/S0040-4039(01)94623-4

4. Castelles, J.; Pujol, F .; Leitjos, H.; Morenomanas, M. Tetrahedron 1982, 38, 337.

http://dx.doi.org/10.1016/0040-4020(82)80170-1

5. Balkrishana, S. B.; Wayne, E. C.; Harold, W. P. Tetrahedron 1981, 37, 2091.

http://dx.doi.org/10.1016/S0040-4020(01)97963-3

6. Tatsuya, S.; Yoshihirio, M.; Jue, H.; Kenji, I,; Fumiaki, I.; Takai, I. J. Org. Chem. 1985, 50, 4967.

http://dx.doi.org/10.1021/jo00224a065

7. Moriuchi, T.; Yamaguchi, M.; KiKushima, K.; Hirao, T. Tetrahedron Lett. 2007, 48, 26672670. 


\section{http://dx.doi.org/10.1016/j.tetlet.2007.02.074}

8. Espenson, J. H.; Zhu, Z.; Zauche, T. H. J. Org. Chem. 1999, 64, 1191. http://dx.doi.org/10.1021/jo9817164

9. Karade, N. N.; Budhewar, V. K.; Katkar, A. N.; Tiwari, G. B. Arkivoc 2006 (xi) 162-167; This paper reports oxidative transformation of aldehydes into methyl esters using diacetoxyiodobenzene in combination with iodine

10. Varvoglis, A. Hypervalent Iodine in Organic Syntheses, Academic: London, 1997.

11. Zhadankin, V. V., Hypervalent Iodine Chemistry, Wiley: Chichester, 2014.

12. Wirth, T.; Hypervalent Iodine Chemistry: Topics in Current Chemistry, Vol. 224, Springer: Berlin, 2003.

13. Zhadankin, V. V., Hypervalent iodine(III) reagents in organic synthesis, Arkivoc 2009 (i) 162.

14. S. Y. Mekhman; , V. M. Andrey; and Zhadankin, V. V., Iodonium salts in organic synthesis, Arkivoc 2011 (i) 370-409.

15. Singh, F. V.; Wirth, T. Chem. Asian J. 2014, 9, 950.

http://dx.doi.org/10.1002/asia.201301582

16. Parra, A.; Reboredo, S. Chem. Eur. J. 2013, 19, 17244.

http://dx.doi.org/10.1002/chem.201302220

17. Moriarty, R. M. J. Org. Chem. 2005, 70, 2893.

http://dx.doi.org/10.1021/jo050117b

18. Moriarty, R. M.; Prakash, O. Org. React. 1999, 54, 273-418.

http://dx.doi.org/10.1002/0471264180.or054.02

19. Moriarty, R. M.; Prakash, O. "Hypervalent Iodine in Organic Synthesis” Acc. Chem. Res. 1986, 19, 244-250.

http://dx.doi.org/10.1021/ar00128a003

20. Furniss, B.; Hannaford, A.J. Vogel's Textbook of Practical Organic Chemistry, $5^{\text {th }}$ Edition, 1989, 869-870.

21. Lucas, H. J.; Kennedy, E. R. Org. Synth., Coll. Vol. IV, John Wiley Sons, Inc., New York, 1955, 355, 482.

22. Sharefkin, J. G.; Saltzman, H. Org. Synth. 1963, 43, 60.

http://dx.doi.org/10.15227/orgsyn.043.0060

23. Karele, B.; Neiland, O. Latv. PSR Zinat. Akad., Vestis, Khim. Ser. 1970, 587; Chem. Abstr. 1971, 74, 42033.

24. Tohma, H.; Maegawa, T.; Kita, Y. Synlett 2003, 5, 723.

25. Tohma, H.; Takizawa, S.; Maegawa, T.; Kita, Y. Angew. Chem. Int. Ed. 2000, 39, 1306. http://dx.doi.org/10.1002/(SICI)1521-3773(20000403)39:7<1306::AIDANIE1306>3.0.CO;2-J

26. Tohma, H.; Takizawa, S.; Maegawa, T.; Kita, Y. Adv. Synth. Catal. 2002, 344, 328. http://dx.doi.org/10.1002/1615-4169(200206)344:3/4<328::AID-ADSC328>3.0.CO;2-S

27. Aldrich, 'Catalog handbook of fine chemicals'. 1990. 
28. Khusnutdinov, R. I.; Shchdneva, N. A.; Bayguzina, A. R.; Mayakova, Y. Y.; Anton, A. S.; Rita, Y. B.; Dzhemilev, U. M. Arkivoc 2004, (xi), 53. 